\title{
Recovery of the tonal hierarchy: Some comparisons across age and levels of musical experience
}

\author{
LOLA L. CUDDY and BETSY BADERTSCHER \\ Queen's University at Kingston, Kingston, Ontario, Canada
}

\begin{abstract}
Two experiments examined the recovery of the tonal hierarchy from three melodic patternsthe major triad, the major scale, and the diminished triad. In the probe-tone technique, for each pattern, each of the 12 tones of the chromatic scale was rated as a completion note for the pattern. Pattern tones and probe tones were synthetic complexes of octave partials, amplitudeweighted according to Shepard (1964). First- through sixth-grade children participated in the first experiment, adults with three levels of musical experience in the second. For all subjects, the probe-tone ratings for the major-triad pattern indicated recovery of the full tonal hierarchy. For the major-scale pattern, children and adults successfully differentiated tonal function within the scale. Adults, however, showed greater sensitivity to key organization than did the children and were less influenced by pitch proximity. The diminished-triad pattern conveyed no musical meaning to the children and was tonally ambiguous for the adults. The importance of the major triad in establishing a sense of key is underscored. As patterns depart from this prototype, recovery of the tonal hierarchy may depend on the degree to which musical knowledge (intuitive and formal) is applied.
\end{abstract}

This paper explores listeners' abstraction of the pitch structures conveyed or implied by melodic patterns in tonal music. It examines the extent to which the tonal hierarchy described by music theory and perceptual experiments may be recovered from different melodic patterns. Adults and first- through sixth-grade children with three levels of musical experience were tested.

It is generally agreed that the composition and appreciation of "tonality," although an extraordinarily complex human activity in its fullest expression (Browne, 1981), involves a hierarchically organized system of pitch relationships. Basic features of the system can be found in standard music texts (e.g., Piston, 1962; Ratner, 1962) and their psychological counterparts in recent theoretical treatments (e.g., Bharucha, 1984; Deutsch \& Feroe, 1981; Dowling, 1978; Krumhansl, 1983; Shepard, 1982). The system is structured at several interrelated levels. First, descriptions of pitch relationships usually include the notion of determinate pitch sets and the assignment

\footnotetext{
We thank C. L. Krumhansl, Cornell University, for discussions, advice, and encouragement. We also thank the Frontenac County Board of Education and the staff of Lord Strathcona Elementary School, especially R. Galbraith and John Gallienne, for permission to test their students and for kind cooperation throughout the study. Daniel Scheidt and Lise de Kok provided cheerful and invaluable research assistance. The data were previously reported in an honors thesis submitted to the Department of Psychology, Queen's University, by Betsy Badertscher. Research was supported by an operating grant from the Natural Sciences and Engineering Council of Canada and an award from the Advisory Research Committee of Queen's University. Requests for reprints should be sent to L. L. Cuddy, Department of Psychology, Queen's University, Kingston, Ontario K7L 3N6, Canada.
}

of specific functions to members of the pitch set. These functions denote, among other things, "stable" and "unstable" notes of the set. Stable and unstable notes differ in their relative frequency and duration within a musical piece and also in the tendency of unstable notes to resolve to stable notes. Second, individual members of the pitch set combine to form simultaneous combinations or chords. Chords may also be arranged in a hierarchy of interchord relations and functions. Finally, at a higher level of abstraction, pitch sets relate to one another in terms of the distance between their tonal centers or points of greatest stability.

We will now briefly elaborate some of the musical terms and notions that underlie the concept of the tonal hierarchy. This material will necessarily be selective; its primary purpose is to introduce the experimental background from which the present research is derived. The musical descriptions will be followed by a consideration of their psychological reality.

The pitch set commonly used in Western tonal music is obtained from the division of the octave into 12 steps called semitones. A semitone step is a frequency increment of about $6 \%$; semitone steps are therefore physically equivalent on a logarithmic scale of frequency. These 12 steps may be represented in a circular projection that wraps around at the octave. This representation, shown at the top of Figure 1, is historically known as the chroma circle. Pitch height, that is, the up versus down dimension of pitch, may be thought of as a dimension perpendicular to the circle. (See Shepard, 1982, for a review of early multidimensional models of musical pitch, models that separated chroma from pitch height.) 


\section{CHROMA CIRCLE}

(FIRST PARTIAL)

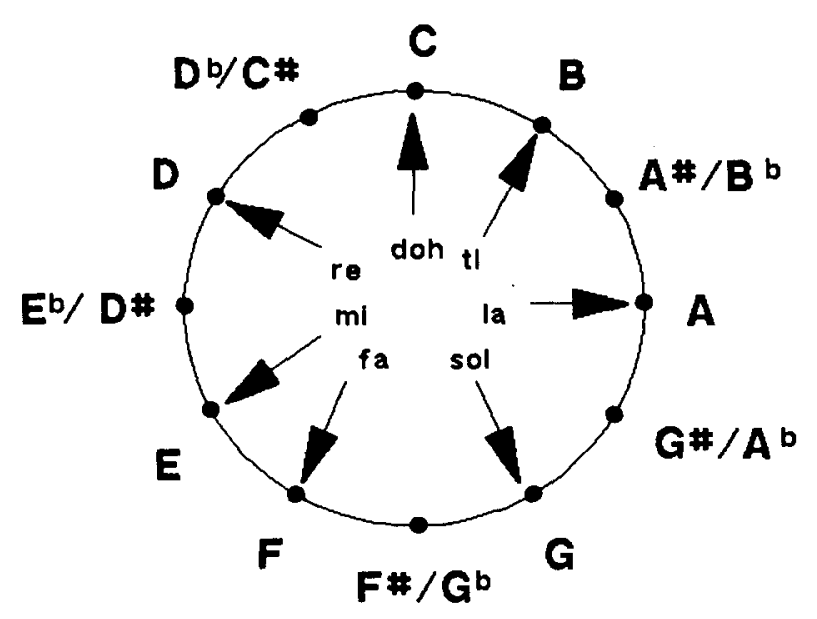

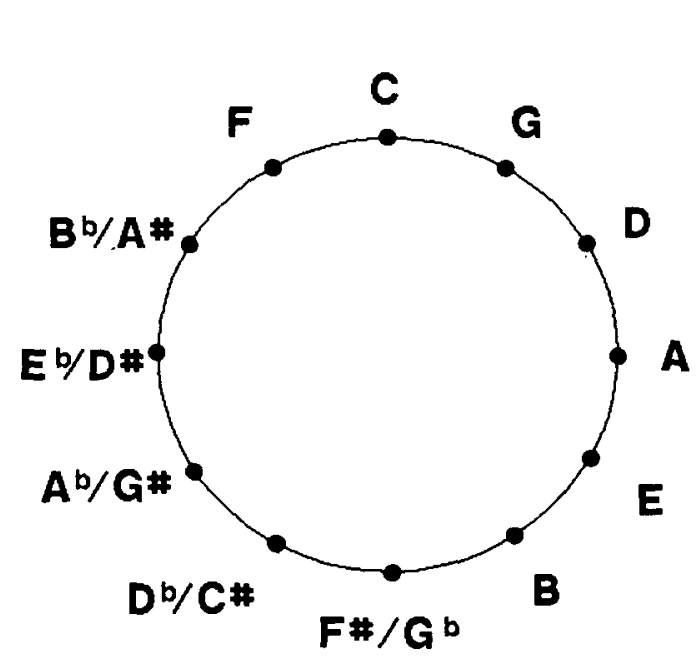

CIRLE OF FIFTHS

(FIFTH PARTIAL)

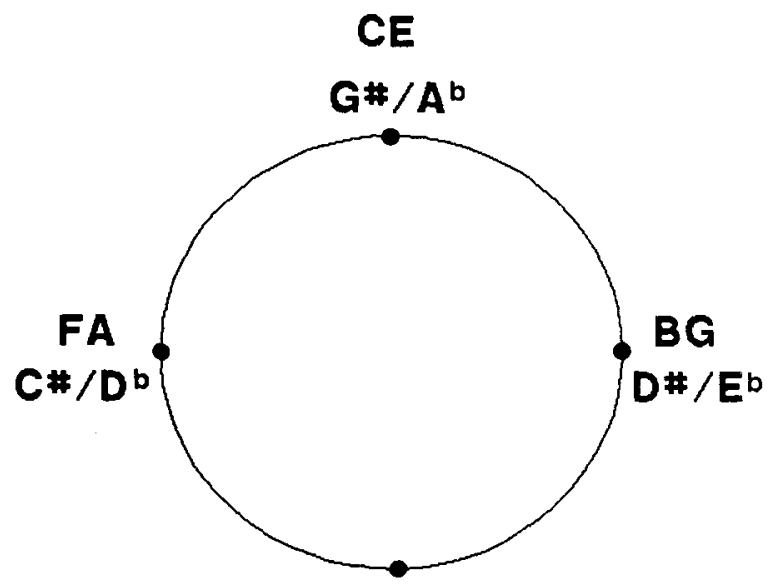

$A \# / B^{b}$

$F \# / G b$
CIRCLE OF THIRDS

(THIRD PARTIAL)

Figure 1. Chroma circle, circle of thirds, and circle of fifths (after Krumhansl, 1982, with permission of the author).

The diatonic scale set is a particular selection of 7 steps on the chroma circle. It is an unequal-interval scale and, as such, possesses some interesting mathematical properties (Balzano, 1982, 1986; Krumhansl, 1986). A tally of all musical intervals, adjacent or nonadjacent, contained in the set reveals the presence of 6 intervals, each of different width. These 6 intervals are the minor second ( 1 semitone), the major second ( 2 semitones), the minor third
( 3 semitones), the major third (4 semitones), the perfect fourth (5 semitones), and the tritone ( 6 semitones). These intervals also have octave complements obtained by subtracting the interval size from 12 semitones; an interval and its octave complement are treated as equivalent. The relative frequency of occurrence of each interval in the set is given by the vector $\langle 2,5,4,3,6,1\rangle$. Note that each interval occurs at least once and occurs a unique 
number of times within the set. Some theorists propose these properties to be critical to the perception of structure in the set (H. Brown \& Butler, 1981; Browne, 1981; Butler \& H. Brown, 1984). According to Browne (1981), rare intervals (the tritone and minor seconds) aid in "position finding" and common intervals (such as the perfect fourth and fifth) aid in "pattern matching." A position-matching task asks for a location in the diatonic "field"; a pattern-matching task asks for judgments of similarity, or same-difference, between patterns of intervals.

The major scale-doh, re, mi, fa, sol, la, ti, doh-is a common example of the diatonic set. The scale is oriented so that, of the two instances of a semitone (first entry in the vector), one occurs between the third and fourth degree of the scale and the other between the seventh and eighth degree. All other intervals between adjacent scale notes are two semitones, also called whole tones. In Figure 1, the notes of the $\mathrm{C}$ major scale are indicated by arrows on the chroma circle, rotating counterclockwise. In the key of $\mathrm{C}$, the semitone steps occur between $\mathrm{E}$ and $\mathrm{F}$, and between $\mathrm{B}$ and $\mathrm{C}$. According to the conventions of music theory, the most stable note of the major scale set is the tonic note, doh, around which are organized the next most stable notes, the notes of the tonic triad, $\mathrm{mi}$ and sol. The remaining diatonic notes are the least stable within the tonality, but are nevertheless in closer relationship to the tonic note than are notes outside the scale set, the nondiatonic notes.

Other cyclic arrangements may be derived from the chroma circle. Two are shown at the bottom of Figure 1. Figure 1 shows a circle of thirds and a circle of fifths adapted from Krumhansl (1982); other theoretical treatments have also described cyclic properties of thirds and fifths (e.g., Balzano, 1986; Longuet-Higgins \& Steedman, 1971; Makeig, 1982; Shepard, 1982; Schoenberg, 1954/ 1969). If the chroma circle is spun clockwise so that it wraps around three times within the octave, the resulting projection is the circle of thirds shown in the lower right of Figure 1. If it is spun clockwise so that it wraps around five times within the octave, the resulting projection is the circle of fifths shown in the lower left of Figure 1. These projections are important because they represent not only or merely relations among single notes, but also relations among tonic notes or keys. In the circle of thirds, tonic notes at the distance of a major third are located at identical points on the circumference. Each tonic note is then flanked by a key note one minor third above on the clockwise side and one minor third below on the counterclockwise side. In the circle of fifths, each tonic note is flanked by the keys sharing all but one constituent member of the scale set. The musical distance between a key and its clockwise neighbor is the interval of a fifth, and the key lies a musical fifth above its neighbor on the counterclockwise side. (It should be noted that this description can readily be extended to relationships between minor keys and between major and minor keys; see, for example, Krumhansl \& Kessler, 1982. These rela- tionships, although important for a complete picture, need not be detailed at this point.)

There is considerable experimental support for the psychological reality of the tonal organization of musical pitch. Studies of recognition memory for short melodic tone sequences have shown that tonal organization facilitates recognition and that the extent of the harm done by the breaking of tonal rules depends on the number of rules broken (Cuddy, Cohen, \& Mewhort, 1981; Cuddy, Cohen, \& Miller, 1979; Dewar, 1974; Dewar, Cuddy, \& Mewhort, 1977; Francès, 1958). On the other hand, an invariance of tonal properties can sometimes fool listeners into thinking a melody has not changed when in fact it has. Dowling (1978) reported that a tonal imitation of a melody, retaining the tonal key of the original melody although altering some interval sizes, is difficult to discriminate from an exact transposition of the melody, which preserves the original interval sizes. However, an atonal imitation-one that introduces nondiatonic notes-is much more readily discriminated from an exact transposition.

Other evidence has been uncovered by the probe-tone technique. A listener is presented with a musical context followed by a probe tone, one of the 12 notes of the chromatic scale. The listener is asked to rate how well the probe fits the context in a musical sense. In a variant of the technique, two probes are presented and the listener is asked to rate how well the second probe follows the first with reference to the musical context. Musical contexts studied include the ascending or descending scale (Krumhansl, 1979; Krumhansl \& Kessler, 1982; Krumhansl \& Shepard, 1979), the notes of the tonic triad as a melodic pattern (Krumhansl \& Keil, 1982), harmonic chords (Krumhansl, 1979; Krumhansl \& Kessler, 1982), and key-defining chord progressions, called cadences (Krumhansl \& Kessler, 1982). All of these contexts yielded a hierarchy of preferences for the probe tones. In single-probe studies, the highest rating was given to a single referent, the tonic note. This note was followed by the notes in the tonic triad, then by notes of the diatonic scale, and, finally, by the nondiatonic notes. Using the two-probe paradigm, Krumhansl (1979) found a directional asymmetry characteristic of psychological hierarchies in other domains (Rosch, 1975). Probe-tone pairs in which the order was an unstable note followed by a stable note were rated higher than were pairs in the reverse order. This asymmetry presumably reflects tendencies in tonal music to resolve unstable tones by following them with stable tones.

It is not entirely clear to what extent the availability of the tonal hierarchy varies with the listener's age and level of musical training. Two studies suggest that the tonal hierarchy cannot be recovered fully from musically untrained adults (Krumhansl \& Shepard, 1979) or from children as young as 6 or 7 (Krumhansl \& Keil, 1982). Krumhansl and Shepard (1979) asked adult listeners to indicate preference for each of 12 chromatic probe tones as completions of the major scale. The probe tone was 
always located in the octave above an ascending major scale or in the octave below a descending major scale. Musically trained listeners preferred completion notes that were stable tones in the tonal hierarchy. However, judgments from musically untrained listeners were largely governed by the factor of pitch height: the nearer in pitch the probe tone was to the notes of the scale passage, the higher it was rated as a completion. Krumhansl and Keil (1982) asked children in first through sixth grade to judge the "goodness" of a six-note melodic pattern. The initial four notes of the pattern were the major triad notes C-E-C-G. The two final notes were probe tones that varied across trials and were located 1 octave from the initial triad. In this case, the only distinction made by the youngest children was that between diatonic and nondiatonic notes. Older children (third and fourth graders) distinguished triad notes from notes not in the triad; only adult subjects isolated the tonic note for highest preference. An analysis of diatonic trials showed that all subjects gave increasingly lower ratings as the scale-step distance between the two probe tones increased.

These two studies may reflect differences in the degree of influence various tonal features exert on the judgments of children and adults. However, given the differences in musical context and in the nature of the probe task, comparisons may not be warranted. More recently, Speer and Meeks (1985) reported greater success in recovering the tonal hierarchy from young children. Second and fifth graders were presented with two scale contextsascending and descending-followed by a single probe tone separated from the context by one half-beat. Speer and Meeks deemed the simpler and shorter single-probe method more appropriate for children. The probe tone was located in the octave above the ascending scale or in the octave below the descending scale. The rating scale was reduced from the original 7-point scale of Krumhansl and Keil (1982) to a 5-point scale. Under these conditions, it was found that, in the descending scale context, all children produced the hierarchical distinctions previously reported for musically sophisticated adults. For the ascending context, the children performed almost as well: all distinguished the tonic note from the notes of the triad, and the triad notes from other diatonic notes. The youngest children failed to distinguish between diatonic and nondiatonic notes on ascending trials; the tendency to rate notes farthest from the scale as poor completions seems to have masked this discrimination.

The present study sought to compare child and adult performance by using the same paradigm and stimuli. The single-probe technique advocated by Speer and Meeks (1985) was used. In addition, both musical contexts and probe tones were generated with spectral characteristics intended to reduce the salience of the pitch-height dimension. Each tone was a synthetic complex of partials separated by octaves, with the amplitude of each partial determined according to the bell-shaped function described by Shepard (1964). The resulting tones possessed chroma but no clearly defined pitch height. The context and the probe were therefore obtained from the same pool of tonal materials, and the potential difficulty of transposing the probe to the octave location of the context was obviated.

The present experiments also compared the probe-tone ratings obtained from three different melodic patterns to evaluate the efficacy of each pattern in producing a sense of key. The melodic patterns tested for evidence of key attribution and key affinity were the major triad, the major ascending scale, and the diminished triad. Certain considerations predict that the major triad pattern should yield a strong sense of a major key. From music theory, possibly the strongest arguments come from Schenker (1906/1954) and theorists in this tradition. For Schenker, the major triad is the prototype for tonal structure, and he demonstrated a means of deriving the diatonic and chromatic system from this prototype (M. Brown, 1986). According to Terhardt's (1984) model of central pitch processing, the major triad is the only triadic chord that unambiguously provides a sense of its fundamental or root note. A temporal-coding model (Patterson, 1986) suggests an alternative mechanism for the extraction of the frequency patterns contained in the major triad, but concurs that diatonicism can be generated from this simple pattern. Krumhansl and Kessler (1982) confirmed empirically that a single harmonically presented triad most strongly evoked the key of which it was the tonic chord.

Thus, if the listener integrates melodic information over time, it might be expected that the melodic triad will evoke a similarly strong sense of the key of its root. Yet in terms of key definition, the major triad is ambiguous. It consists of 2 intervals of a third, a minor third stacked on a major third. The top and bottom note form the interval of a fifth. From the vector containing the relative frequency of occurrence of these intervals within a diatonic major scale, it can be established that no component interval unambiguously defines a single key. There are four minor thirds, three major thirds, and six perfect fifths (octave complement of the fourth) in the major scale. The particular combination of the intervals contained in the major triad can occur three times in a major scale-on the first, the fourth, and the fifth scale degree. The major triad pattern C-E-C-G, for example, can occur in the keys of $\mathrm{C}, \mathrm{F}$, and $\mathrm{G}$ major. Thus, the evocation of one key by default may represent an overlearned strategy employed only by the musically sophisticated. Speer and Meeks (1985) questioned whether the melodic triad is a suitable context for establishing the tonal hierarchy with young children.

The major-scale pattern, on the other hand, uniquely defines a major key through its component intervals. It contains the elements of the major triad, but in addition contains both common and rare intervals. The stimulus itself not only begins on the tonic note but also cues the special locations of the semitone intervals and the tritone that is unique to its key. For Schenker (1906/1954), however, the major scale is derived from the major triad; as noted above, the major triad, not the diatonic scale, is 
the basic element on which the tonal hierarchy is built. This claim and its implications, according to $\mathbf{M}$. Brown (1986), separate Schenker from 19th-century traditions in music theory.

The third pattern tested, the diminished triad, also uniquely defines a major key. Unlike the major scale, it contains only three different notes, and, unlike the major triad, it is psychoacoustically dissonant, with an ambiguous root. Howevẹr, it contains intervallic information that logically defines a tonic note. The diminished triad consists of two stacked minor thirds, which together form the interval of a tritone. This pattern of intervals captures only one diatonic major set; the diminished triad occurs only on the seventh degree of the major scale. Moreover, the diminished triad contains two scale degrees, the fourth and the seventh (fa and ti, respectively), which in tonal music produce strong tendencies or expectations to resolve to the stable notes of the tonic triad ( $\mathrm{mi}$ and doh, respectively). H. Brown and Butler (1981), following Browne's (1981) notion that rare intervals aid in position finding, argued that the tritone plays a unique and unambiguous role in locating the tonal center of a melodic passage. In support, they report experimental results showing that musically trained listeners located the tonic of three-note patterns containing the tritone with ease, and almost as accurately as they located any one of the tonics of "multivalent" patterns, that is, diatonic patterns not containing the tritone. None of the "correct" tonics for the multivalent patterns was chosen more than $44 \%$ of the time, compared with $91 \%$ accuracy for the correct tonic of the tritone patterns. Data for the major triad patterns were not presented separately from other multivalent patterns, so it is not possible to evaluate the degree of superiority of the tritone patterns over triadic patterns. The authors stated, however, that triadic and scalar constructs "cannot be considered reliable context-independent indicators of tonality" (H. Brown \& Butler, 1981, p. 48).

Thus, in addition to the initial purpose of comparing the recovery of the tonal hierarchy at different levels of age and experience, we planned to obtain tonality profiles for each of three patterns and to compare the tonal structures recovered from the profiles.

\section{EXPERIMENT 1}

In the first experiment, tonality profiles were obtained from elementary school children, aged 6 to 12 , for each of three musical contexts-the major triad, the major ascending scale, and the diminished triad.

\section{Method}

Subjects. The subjects were 53 elementary school children, 27 male and 26 female. Twenty were recruited from first- and second grade classes, 21 from third and fourth grade, and 12 from fifth and sixth grade. Parental consent was obtained prior to testing, and the parents also provided information on the children's musical backgrounds. All of the subjects attended regular music classes at the school, about $1.5 \mathrm{~h}$ per week.
The first and second graders ranged in age from 6.3 years to 8.1 years, with a mean age of 7.0 years. Nine had taken private music lessons for an average of 1.0 year. The third and fourth graders ranged in age from 8.2 years to 10.6 years, with a mean age of 9.0 years. Nine had taken private music lessons for an average of 1.5 years. The fifth- and sixth-grade children ranged in age from 9.3 years to 12.5 years, with a mean age of 10.9 years. Five were studying music privately, with an average of 1.8 years of instruction.

Apparatus. Stimuli were digitally synthesized by a DMX-1000 signal processor (Wallraff, 1979) under control of a PDP 11/23 host computer. The sampling rate was $19.3 \mathrm{kHz}$. Each stimulus tone consisted of nine sine-wave components spaced at octave intervals within the range $16 \mathrm{~Hz}$ to $8000 \mathrm{~Hz}$. The amplitudes of the components were selected according to a cosine-shaped spectral envelope with peak amplitude at F:4 $(370 \mathrm{~Hz})$. Rise time and fall time for each tone were each $25 \mathrm{msec}$. Frequency values for the tones were determined according to the system of equal temperament with $A 4=440 \mathrm{~Hz}$. Tones were recorded on cassette tape (TDK D90) by a stereo cassette recorder (Alpine AL-35).

Three different sequential tone patterns were constructed, based on either the major triad, the major scale, or the diminished triad. In the key of $C$, these patterns corresponded to the notes $C-E-C-G$, C-D-E-F-G-A-B-C, and B-D-B-F, respectively. On each trial for a given pattern, the tones of the pattern were followed by a single probe tone, one of the 12 possible notes of the chromatic scale. Duration of each tone of the pattern was $0.33 \mathrm{sec}$; duration of the probe tone was $1.00 \mathrm{sec}$. The probe tone was separated from the last tone of the sequence by an interval of $0.1 \mathrm{sec}$ (cf. Speer \& Meeks, 1985). This gap between the end of the pattern and the probe tone appeared to place a very slight rhythmic emphasis on the probe tone.

For each pattern, six different blocks of trials were recorded. Each block contained each of the 12 different probe tones paired once with the pattern, for a total of 12 trials. Order of probe tones was randomized within the block. Each pattern within each block was randomly assigned to one of three frequency locations; the first note was either $B, C$, or $C$, and the remaining tones of the pattern and the probe tone were transposed accordingly.

Procedure. Each child received three blocks of trials, one for each of the three tone patterns. Each block was randomly selected from the six prepared for a given pattern. The order of the patterns was randomized across subjects. The trials were reproduced through the speakers of a JVC RC-363JW/C stereo cassette recorder at a comfortable loudness level.

Each session began with the explanation that the experimenter was trying to write a song and needed help with the ending. The subjects were asked to listen to each trial and to rate whether it sounded "good" or "bad." They were shown a rating scale and were told that they could respond either by choosing 1 of 7 dots along a line, or by verbally indicating the corresponding number. Over the rightmost dot (1) was a smiling face; over the middle dot, (4), a neutral face; and over the leftmost dot (7), was a frowning face. The subjects were encouraged to use the full range of the scale.

The subjects were tested individually. Each session began with sufficient practice trials, played on a piano by the experimenter, to allow the subject to feel comfortable with the task. Most subjects required only three or four practice trials, and no one required more than eight. The subjects heard and judged the practice and then the experimental trials. Sessions lasted approximately $20 \mathrm{~min}$.

\section{Results and Discussion}

Overall analysis. Following Krumhansl and Kessler (1982), the set of 12 probe-tone judgments for each pattern will be called the profile for that pattern. First, all 
Table 1

Average Probe-Tone Ratings for Three Tone Patterns and Three Grade Levels

\begin{tabular}{|c|c|c|c|c|c|c|c|c|c|c|c|c|}
\hline \multirow[b]{2}{*}{ Grade } & \multicolumn{12}{|c|}{ Probe Tone } \\
\hline & $\mathrm{C}$ & $\mathrm{C}$ & $\mathrm{D}$ & $\mathrm{D}$ & $\mathbf{E}$ & $\mathrm{F}$ & $\mathrm{FH}$ & $\mathrm{G}$ & $\mathrm{G}$ & A & $A$ & $\mathbf{B}$ \\
\hline \multicolumn{13}{|c|}{ Major Triad } \\
\hline $1-2$ & 1.60 & 3.80 & 2.95 & 3.90 & 2.90 & 3.45 & 3.90 & 2.70 & 3.55 & 3.05 & 3.55 & 3.80 \\
\hline $3-4$ & 1.91 & 3.62 & 3.52 & 3.91 & 3.05 & 2.86 & 3.19 & 2.62 & 4.05 & 2.81 & 3.81 & 3.91 \\
\hline $5-6$ & 2.25 & 4.25 & 4.42 & 5.08 & 3.50 & 3.25 & 4.83 & 3.75 & 4.42 & 3.92 & 5.08 & 3.75 \\
\hline \multicolumn{13}{|c|}{ Major Scale } \\
\hline $1-2$ & 1.25 & 3.10 & 4.05 & 3.95 & 3.85 & 4.50 & 4.05 & 2.85 & 4.05 & 3.75 & 4.30 & 2.85 \\
\hline $3-4$ & 1.29 & 2.62 & 4.05 & 4.33 & 3.91 & 4.19 & 4.43 & 3.86 & 4.62 & 4.52 & 3.95 & 2.86 \\
\hline $5-6$ & 1.50 & 5.00 & 5.50 & 6.00 & 4.83 & 4.50 & 5.08 & 3.33 & 5.42 & 4.25 & 5.33 & 4.50 \\
\hline \multicolumn{13}{|c|}{ Diminished Triad } \\
\hline $1-2$ & 2.45 & 3.95 & 3.95 & 3.40 & 3.55 & 3.00 & 3.00 & 3.05 & 3.00 & 3.75 & 3.15 & 2.85 \\
\hline $3-4$ & 3.71 & 4.24 & 3.29 & 3.71 & 3.62 & 3.00 & 2.91 & 2.86 & 3.62 & 3.43 & 3.43 & 3.62 \\
\hline $5-6$ & 3.50 & 4.33 & 3.75 & 3.42 & 4.08 & 3.50 & 3.92 & 4.25 & 3.75 & 3.58 & 3.92 & 4.25 \\
\hline
\end{tabular}

probe-tone judgments were recorded with respect to the interval between the first note of the pattern and the probe note. They were then aligned with respect to the key of $\mathrm{C}$, that is, as if the first note of the triad and the scale pattern were always $C$, and as if the diminished pattern were always B-D-B-F. For all profiles, therefore, the probe-tone levels ran through the chromatic scale from $\mathrm{C}$ to $\mathrm{B}$, with $\mathrm{C}$ as the correct tonic note for each profile.

The average probe-tone ratings for each pattern for each grade classification are given in Table 1 . For each pattern, the profiles reflected a similar trend at each grade level; that is, children at all grade levels responded to each pattern in the same way. The profiles for each pattern, averaged across the three grade levels, are shown in Figure 2. Here there are visible differences among the patterns, to be described in detail below.

The data were next subjected to an overall analysis of variance with one between-subjects factor, school grade (3 levels), and two within-subject factors, patterns (3 levels) and probe tones ( 12 levels). For the analysis, the data were normalized by converting each response to a standard-deviation score about the subject's own mean rating (see Krumhansl \& Keil, 1982). The interaction between grade, pattern, and probe tone was not significant $[F(44,1100)=1.07]$.

Two additional analyses were conducted: One dropped the 7 children with more than 2 years' musical training from the analysis; the other reassigned children to three equal-sized groups according to age. Again, no statistically significant effects attributable to group assignment emerged. The interaction between patterns and probe tones, however, was significant $[F(22,1100)=6.02$, $p<.001]$, and the profile for each pattern was subjected to further analyses.

Profile analysis-orthogonal contrasts. For the major triad pattern, shown in the top panel of Figure 2, the highest rating was given to the tonic note, $\mathrm{C}$. The tonic note was rated higher than the dominant note, $\mathrm{G}[F(1,50)$ $=20.39, p<.0001]$. The ratings for $\mathrm{C}$ and $\mathrm{G}$ averaged together were higher than the ratings for the remaining note of the triad, the mediant, $\mathrm{E}[F(1,50)=8.67$, $p<.01]$. Ratings for triad notes were higher than ratings for diatonic notes not in the triad, F, D, A, and B $[F(1,50)=26.84, p<.0001]$. Finally, diatonic notes were rated higher than nondiatonic notes $[F(1,50)=$ $42.79, p<.0001]$. The correlation between the profile
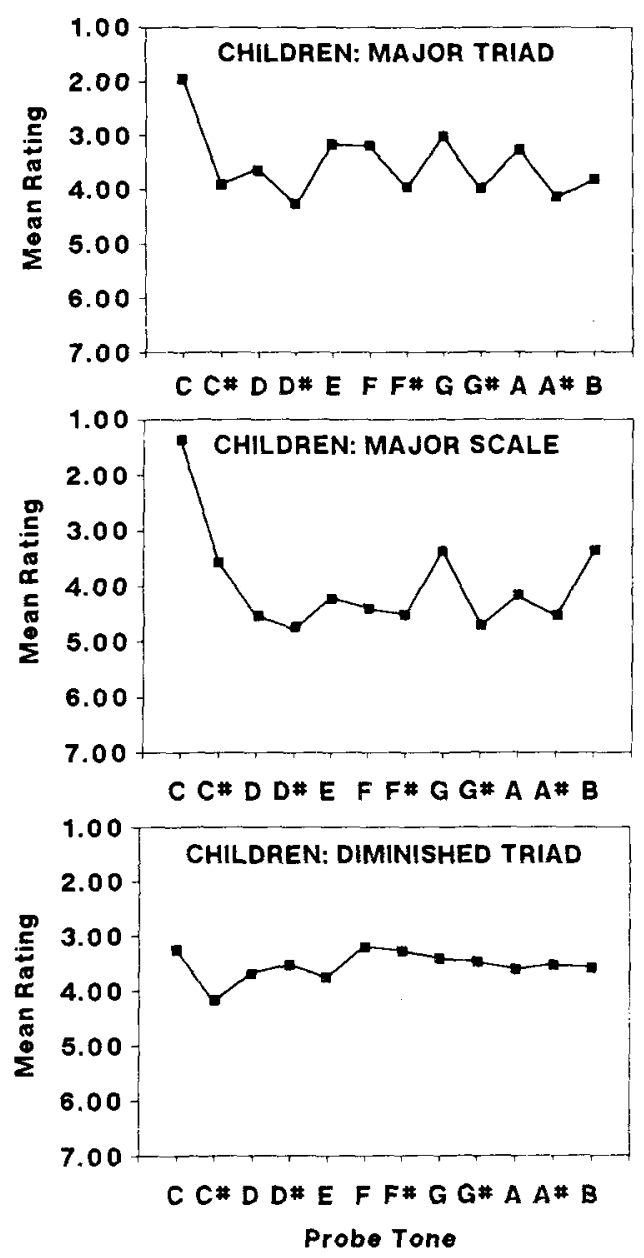

Figure 2. Children's average probe-tone ratings for the major triad, major scale, and diminished triad. 
ratings for the major triad and the Krumhansl and Kessler (1982) C major profile was 0.97 , significant beyond the .01 level.

The profile for the scale pattern is shown in the middle panel of Figure 2. There is again a marked preference for the tonic note, and other effects were also similar to those found for the major triad profile. The tonic note was rated higher than the dominant note $[F(1,50)=70.08$, $p<.00011$; the tonic and dominant notes were rated higher than the mediant $[F(1,50)=79.71, p<.0001]$; notes from the triad were rated higher than diatonic notes not in the triad $[F(1,50)=44.81, p<.0001]$; and diatonic notes were rated higher than nondiatonic notes $[F(1,50)=36.45, p<.0001]$. The scale data differ from the triad data, however, in that they reveal an additional preference for notes falling near the tonic on the chroma circle. As can be seen in the figure, the notes $C \sharp$ and $B$ are rated as high as the dominant, $\mathrm{G}$, and are second only to the tonic note, $\mathrm{C}$. Within the set of orthogonal contrasts, the probe-tone rating for $\mathrm{B}$ was found to be significantly higher than that for $\mathrm{A}[F(1,50)=8.61$, $p<.01]$, and the rating for $\mathrm{C}$ was significantly higher than that for $\mathrm{D}[F(1,50)=16.54, p<.001]$. The correlation between the profile for the major scale and the Krumhansl and Kessler (1982) C major profile was 0.73, significant beyond the .01 level.

The profile for the diminished triad pattern is shown in the bottom panel of Figure 2 . It is essentially flat. The main effect of probe tone was not significant, nor, not surprisingly, were any of the contrasts reflecting preference for elements of the tonic triad. Any variation in the profile for the diminished-triad pattern must be attributed to random sources. The correlation between the profile for the diminished-triad pattern and the Krumhansl and Kessler (1982) C major profile, 0.40, was not significant.

Profile analysis-Fourier components. The Fourier analysis of discrete data is a manner of partitioning variance that is conceptually similar to the analysis of variance. It provides an alternative description that is a potentially useful tool for quantifying the amount of tonal structure in a profile. The application of Fourier analysis to probe-tone profiles was first suggested by $\mathrm{H}$. L. Kaplan (personal communication, 1981) and has since been implemented by Krumhansl (1982) and Krumhansl and Schmuckler (1986). Kaplan's argument was that tonal structure in a profile should be revealed in two periodic components, one with three cycles per octave and one with five. These two cycles correspond to the dimensions of the toroidal key-space proposed by Krumhansl and Kessler (1982) to represent the organization of major and minor keys. In the Fourier analysis, the two cycles would be present as the third and fifth partials. Krumhansl (1982), in applying the Fourier analysis to the original profiles reported by Krumhansl and Kessler (1982), did indeed find that the third and fifth partials had the largest amplitudes for both major and minor profiles. She further proposed that the amount of variance in the profile that can be accounted for by these two partials may be used to quantify the "tonal strength" represented by the profile.

The results of the Fourier analysis of the profiles in the present experiment are given in Table 2. The method of analysis was that described by Jenkins and Watts (1968, pp. 17-21). The table shows the amplitudes, phases, and the proportion of variance accounted for for each of six partials. (The zeroth partial is simply the mean of the data set; the value is subtracted from the data before the computations, and so the partial has zero amplitude.)

Note that for the major triad most of the variance in the profile was accounted for by the third and fifth partials. The profile was resynthesized from these two partials, and the correlation between the obtained and resynthesized profiles was $0.89(p<.01)$. Neither of the other two patterns could be resynthesized successfully from these two partials. For the major scale, a large portion of the variance was located in the first and second partials. These partials reflect the overall "scoop" or U and the gently undulating $W$ shape in the profile. The analysis for the diminished triad is not amenable to interpretation, given the instability of the mean probe-tone ratings for this profile.

Summary of findings. The children's profile for the melodic major pattern was virtually identical to the profile for major chords and chord cadences obtained from musically sophisticated adults (Krumhansl \& Kessler, 1982). Sufficient information was present in the profile to implicate the cyclic properties of key structure, and by Krumhansl's (1982) definition of tonal strength, the

Table 2

Fourier Analysis of Children's Profiles for Major Triad, Major Scale, and Diminished Triad

\begin{tabular}{|c|c|c|c|}
\hline Partial & Amplitude & Phase & $\%$ Total Variance \\
\hline \multicolumn{4}{|c|}{ Major Triad } \\
\hline 0 & 4.474 & 0.000 & 0.000 \\
\hline 1 & 0.037 & 355.447 & 0.678 \\
\hline 2 & 0.183 & 185.446 & 16.617 \\
\hline 3 & 0.232 & 343.321 & 26.920 \\
\hline 4 & 0.088 & 158.753 & 3.831 \\
\hline 5 & 0.320 & 37.774 & 51.222 \\
\hline 6 & 0.054 & 180.001 & 0.732 \\
\hline \multicolumn{4}{|c|}{ Major Scale } \\
\hline $\mathbf{0}$ & 4.043 & 0.000 & 0.000 \\
\hline 1 & 0.320 & 11.513 & 24.378 \\
\hline 2 & 0.391 & 175.978 & 36.335 \\
\hline 3 & 0.282 & 10.713 & 18.961 \\
\hline 4 & 0.162 & 142.187 & 6.273 \\
\hline 5 & 0.243 & 34.072 & 14.026 \\
\hline 6 & 0.015 & 359.996 & 0.027 \\
\hline \multicolumn{4}{|c|}{ Diminished Triad } \\
\hline 0 & 4.478 & 0.000 & 0.000 \\
\hline 1 & 0.089 & 152.178 & 23.931 \\
\hline 2 & 0.073 & 222.814 & 16.139 \\
\hline 3 & 0.038 & 90.000 & 4.417 \\
\hline 4 & 0.093 & 227.838 & 25.717 \\
\hline 5 & 0.094 & 345.695 & 26.751 \\
\hline 6 & 0.045 & 180.001 & 3.044 \\
\hline
\end{tabular}


profile may be considered "very strong." The profile for the major scale showed the primacy of the tonic note, followed by the tonic triad and the diatonic notes. However, recovery of tonal structure was partially masked by the influence of pitch proximity on judgment. Although the profile differed in shape from that obtained by Speer and Meeks (1985) for the ascending scale context, the two profiles probably reflect similar influences. Speer and Meeks used flute-stopped organ tones as stimuli; probe tones were rejected as scale completions as they became more distant in pitch from the scale context. In our experiment, the same probe tones, by virtue of their spectral content, would very likely be increasingly closer in perceived pitch to the correct tonic. Thus, in both cases, pitch proximity may have been influential. The essentially flat profile for the diminished triad suggested that this pattern conveyed no musical meaning to the children.

No developmental trends were apparent in this experiment. All children responded similarly to each musical pattern.

\section{EXPERIMENT 2}

In the second experiment, tonality profiles were obtained from young adults (university students) at three levels of musical experience. The musical contexts were the same as in the first experiment.

\section{Method}

Subjects. The subjects were 45 volunteers, 21 male, 24 female, 15 from each of three categories of musical experience. The subjects' ages in each category ranged from 18 to 35 , with an average age of 21.3 years. Subjects in the musically untrained group had less than 2 years of formal instruction; 6 had an average of 15 months of instruction, the rest had none. Those in the musically trained group had an average of 7.5 years of training; 7 had taken Royal Conservatory exams and had achieved, on the average, a Grade V level of performance. Subjects in the highly trained group had attained at least a Grade VII Royal Conservatory level of performance or equivalent in an instrument or voice; they had, on the average, 14.5 years of formal training and had achieved a Grade IX level of performance. (In Ontario, Grade VIII and IX performance is accepted as the practical component for music credit for school grades 12 and 13, respectively [university admission level].)

Apparatus and Procedure. The apparatus and procedure were similar to those of Experiment 1, with the following modifications. Each subject heard two blocks of trials, rather than one, for each pattern, and the order of patterns was counterbalanced across subjects according to a replicated Latin square. Trial blocks were reproduced on a cassette recorder (Alpine AL-35) and delivered binaurally through headphones (Sennheiser HD-424).

Each session began with the explanation that the experimenter was studying music perception and that the subject would be asked to give his or her honest, subjective evaluations of how well the final note of each pattern provided a musical completion to the pattern. The subjects were given a 7-point rating scale to record their responses, with 1 designated sounds good and 7 designated sounds bad. The subjects were asked about their musical backgrounds; then they proceeded to hear and rate 5 practice and 72 experimental trials. Total testing time was 30 to $40 \mathrm{~min}$.

\section{Results and Discussion}

Overall analysis. The design for the overall analysis was similar to that of Experiment 1, with the addition of the counterbalancing factors of the Latin square. The factor of school grade was replaced by musical experience (with three levels). The factors of probe tone and pattern were, of course, the same.

The average probe-tone rating for each pattern and each level of musical experience is shown in Table 3. It was found that the three subject groups responded similarly to each pattern with one exception: the profiles for the highly trained group were more sharply defined. Highly trained subjects expressed greater willingness to use the full rating scale. However, the analysis of normalized data showed that the interaction between pattern, probe tone, and level of musical experience was not significant $[F(44,924)=1.20]$. Differences among profiles for the different patterns were clearly present and led to a significant interaction between pattern and probe tone $[F(22,924)=13.08, p<.001]$. The differences among profiles are discussed below. Finally, it was noted that the Latin square variables of order of patterns (rows) and

Table 3

Average Probe-Tone Rating for Three Tone Patterns and Three Levels of Musical Experience

\begin{tabular}{|c|c|c|c|c|c|c|c|c|c|c|c|c|}
\hline \multirow[b]{2}{*}{ Experience } & \multicolumn{12}{|c|}{ Probe Tone } \\
\hline & $\mathrm{C}$ & $C$ & $\mathbf{D}$ & $\mathrm{D}$ & $\mathrm{E}$ & $\mathbf{F}$ & $\mathrm{FH}$ & $\mathbf{G}$ & GH & $\mathbf{A}$ & A & B \\
\hline \multicolumn{13}{|c|}{ Major Triad } \\
\hline Low & 3.03 & 4.80 & 3.57 & 4.67 & 3.90 & 3.07 & 4.50 & 3.40 & 4.10 & 3.53 & 3.90 & 4.20 \\
\hline Medium & 1.83 & 4.43 & 3.83 & 5.50 & 3.73 & 2.93 & 4.60 & 2.57 & 4.13 & 3.77 & 4.20 & 4.47 \\
\hline High & 1.87 & 4.90 & 3.97 & 4.97 & 3.10 & 3.33 & 4.50 & 2.67 & 4.07 & 3.87 & 4.17 & 4.23 \\
\hline \multicolumn{13}{|c|}{ Major Scale } \\
\hline Low & 2.17 & 4.93 & 4.67 & 5.37 & 4.70 & 4.97 & 5.20 & 4.23 & 5.03 & 4.17 & 5.13 & 3.93 \\
\hline Medium & 1.27 & 5.43 & 5.50 & 5.87 & 4.70 & 4.90 & 5.67 & 3.97 & 5.53 & 4.47 & 5.90 & 4.07 \\
\hline High & 1.60 & 5.60 & 4.90 & 5.77 & 3.93 & 4.80 & 5.00 & 3.40 & 5.73 & 3.90 & 4.97 & 3.30 \\
\hline \multicolumn{13}{|c|}{ Diminished Triad } \\
\hline Low & 3.27 & 4.00 & 3.67 & 3.63 & 4.27 & 4.17 & 3.77 & 4.10 & 4.67 & 4.03 & 3.60 & 3.10 \\
\hline Medium & 3.87 & 4.43 & 4.20 & 4.27 & 2.97 & 3.23 & 3.03 & 4.80 & 4.23 & 4.03 & 3.53 & 2.83 \\
\hline High & 3.63 & 4.63 & 3.43 & 3.93 & 3.33 & 3.23 & 2.80 & 4.03 & 4.00 & 3.87 & 4.17 & 3.27 \\
\hline
\end{tabular}


practice (columns) in Experiment 2 did not approach significance at the .05 level.

Profile analysis -orthogonal contrasts. The profiles for each pattern are shown in Figure 3. Visual comparison of Figures 2 and 3 indicates fair correspondence between the profiles for the same pattern. The similarity was borne out in the statistical analyses. Certain differences, however, were also revealed.

For the major triad (top panel of Figure 3), it was found that the tonic note was preferred over the dominant note $[F(1,42)=19.85, p<.0001]$; the tonic and dominant were together rated higher than the mediant $[F(1,42)=$ $24.98, p<.0001]$; within the diatonic set, triad notes were rated higher than nontriad notes $[F(1,42)=50.62$, $p<.0001]$; and diatonic notes were rated higher than nondiatonic notes $[F(1,42)=69.67, p<.0001]$. There were two significant effects not found in the children's data. The adults rated the subdominant, F, higher than the other nontriad notes of the diatonic set $[F(1,42)=$ $13.04, p<.001]$, and rated the note A (key note of the relative minor) higher than the note $\mathrm{B}[F(1,42)=5.53$, $p<.05]$. Among the nondiatonic notes, no differential
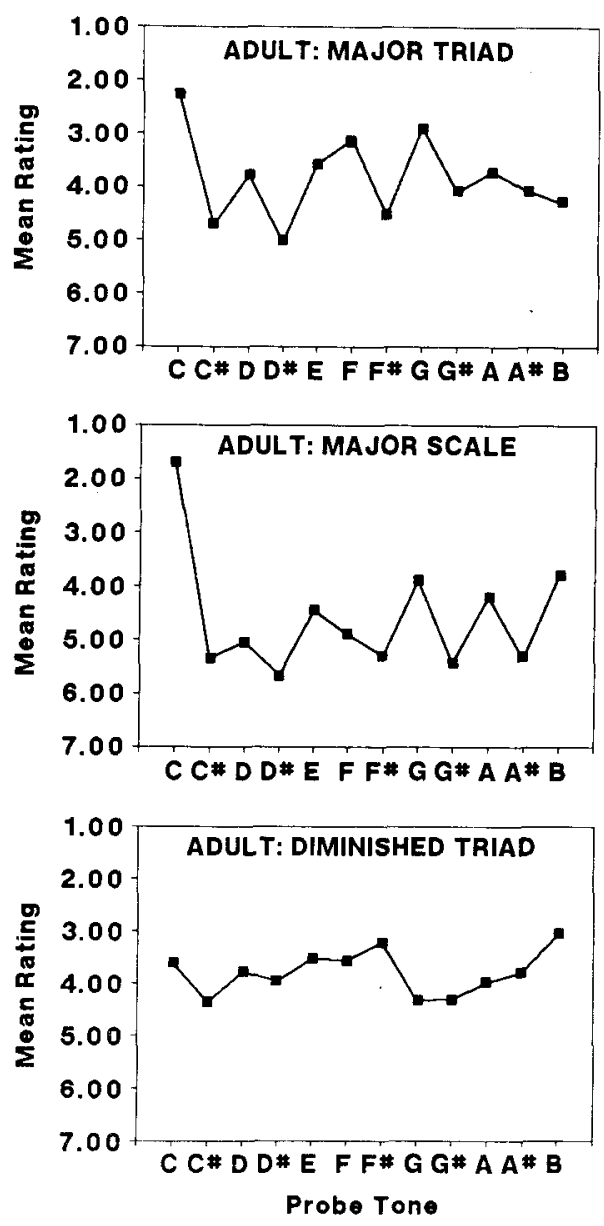

Figure 3. Adult's average probe-tone ratings for the major triad, major scale, and diminished triad. preferences were found. The correlation between the major triad profile and the Krumhansl and Kessler (1982) $\mathrm{C}$ major profile was $0.94(p<.01)$.

For the scale pattern (middle panel of Figure 3), the tonic was rated higher than the dominant note $[F(1,42)$ $=92.66, p<.0001]$; the tonic and dominant together were rated higher than the mediant $[F(1,42)=81.15$, $p<.0001]$; within the diatonic set, triad notes were rated higher than nontriad notes $[F(1,42)=101.48$, $p<.0001]$; and diatonic notes were rated higher than nondiatonic notes $[F(1,42)=173.36, p<.0001]$. These findings were similar to the children's results. Other findings, not present in the children's results, emerged. Adults rated the note $F$ (the subdominant) lower than the average for the other diatonic notes not in the triad $(\mathrm{D}, \mathrm{A}$, and $B)[F(1,42)=15.99, p<.001]$. Although the difference between $A$ and $B$ and between $C \$$ and $D \sharp$ was in the same direction as the differences found in the children's data, the rating for A was not significantly lower than the rating for $\mathrm{B}[F(1,42)=1.81]$. The rating for $\mathrm{C} \sharp$ was, however, significantly higher than the rating for $\mathrm{D} \sharp[F(1,42)=4.12, p<.05]$. The correlation between the major scale profile and the Krumhansl and Kessler (1982) C major profile was $0.85(p<.01)$.

The profile for the diminished triad pattern B-D-B-F is shown in the bottom panel of Figure 3. As was the case with the children, this profile was less well defined than the profiles for the major triad and the major scale. Unlike the children's results, significant effects of varying the probe tone were found. There was a significant preference for the tonic $\mathrm{C}$ over the dominant $\mathrm{G}[F(1,42)=$ $15.36, p<.001]$; for B over A $[F(1,42)=23.96$, $p<.0001]$; and for $F$ over the average for $C \sharp$, $D \sharp$, and $\mathrm{G} \$[F(1,42)=20.25, p<.0001]$. On the average, diatonic notes were rated higher than nondiatonic notes $[F(1,42)=5.03, p<.05]$. The notes of the triad in $\mathrm{C}$ major were not, however, preferred over nontriad notes in the diatonic set. The correlation between the profile for the diminished triad pattern and the Krumhansl and Kessler (1982) C major profile was 0.074.

The diminished triad suggested several satisfactory completions $-\mathrm{C}, \mathrm{F} \$$, and $\mathrm{B}$. This assignment among three probe tones was not attributable to three different types of subject strategy. Subjects tended to respond similarly, and the error term for the analysis-reflecting the subject $X$ probe tone interaction-was similar for all three patterns $(0.46,0.36$, and 0.48 for the major triad, major scale, and diminished triad pattern, respectively).

Profile analysis-Fourier components. The results of the Fourier analysis for each pattern are given in Table 4. For the adult data, both the major triad and the major scale could be successfully reconstituted from the third and the fifth partials. The correlation between the resynthesized and original profiles was 0.91 for the major triad and 0.76 for the major scale. Both correlations were significant beyond the .01 level. The diminished-triad profile could not be resynthesized successfully from the third and fifth partials. Most of the variance resided in the second partial. 
Table 4

Fourier Analysis of Adult Profiles for Major Triad, Major Scale, and Diminished Triad Patterns

\begin{tabular}{|c|c|c|c|}
\hline Partial & Amplitude & Phase & $\%$ Total Variance \\
\hline \multicolumn{4}{|c|}{ Major Triad } \\
\hline 0 & 4.160 & 0.000 & 0.000 \\
\hline 1 & 0.086 & 114.129 & 2.480 \\
\hline 2 & 0.191 & 188.910 & 12.324 \\
\hline 3 & 0.214 & 344.667 & 15.504 \\
\hline 4 & 0.022 & 9.636 & 0.157 \\
\hline 5 & 0.446 & 25.955 & 67.106 \\
\hline 6 & 0.120 & 180.000 & 2.431 \\
\hline \multicolumn{4}{|c|}{ Major Scale } \\
\hline $\mathbf{0}$ & 3.425 & 0.000 & 0.000 \\
\hline 1 & 0.291 & 28.273 & 14.968 \\
\hline 2 & 0.350 & 194.587 & 21.602 \\
\hline 3 & 0.353 & 14.923 & 21.990 \\
\hline 4 & 0.185 & 176.874 & 6.067 \\
\hline 5 & 0.446 & 46.826 & 35.206 \\
\hline 6 & 0.043 & 180.001 & 0.166 \\
\hline \multicolumn{4}{|c|}{ Diminished Triad } \\
\hline 0 & 4.222 & 0.000 & 0.000 \\
\hline 1 & 0.040 & 284.884 & 1.947 \\
\hline 2 & 0.227 & 244.386 & 63.342 \\
\hline 3 & 0.072 & 140.617 & 6.443 \\
\hline 4 & 0.105 & 241.186 & 13.511 \\
\hline 5 & 0.091 & 124.951 & 10.121 \\
\hline 6 & 0.087 & 180.000 & 4.637 \\
\hline
\end{tabular}

Summary of findings. The adult profile for the melodic major pattern was similar to that obtained from the children and to the major-key profile obtained by Krumhansl and Kessler (1982): it was tonally "very strong." The major-scale profile showed that the adults were somewhat less influenced by pitch proximity than were the children. The cyclic properties of key structure were present in the adult major-scale profile. However, the scale was not as effective as the melodic major triad in recovering the tonal hierarchy. The diminished-triad pattern did not recover a profile that could be considered tonal.

Although this pattern has logical, key-defining properties based on interval content, it appears that this information was not abstracted for purposes of producing a tonal hierarchy. Various interpretations of the profile are possible. The simplest is that preferred completions were the top and bottom notes of the pattern plus the notes in close pitch proximity to these two notes. A more abstract, but still plausible, explanation is that the profile is a mixture of tonalities- $\mathrm{C}$ major and the harmonic minor keys that contain the diminished-triad pattern. Two harmonic minor keys, $\mathrm{C}$ minor and $\mathrm{A}$ minor, contain the pattern $B-D-B-F$, and the harmonic minor of $F$ B-E Invoking minor keys produces problems for approaches to tonality that focus exclusively on the interval properties of the diatonic system. Minor keys are not diatonic and do not possess the property of having their key centers uniquely defined by the vector of interval frequencies (Browne, 1981). However, other approaches accord the minor-key system a status similar to that of the majorkey system (e.g., Krumhansl \& Kessler, 1982) and are compatible with the notion that perceived ambiguity may result from several opposing tendencies (see also Cuddy, 1985,1986 ).

The profiles for the different levels of musical experience were essentially similar.

Supplementary experiment. The failure of highly trained listeners to produce a tonal profile for the diminished triad was surprising, in view of the results of H. Brown and Butler (1981) (and our own intuitions from musical experience). Interviews conducted after data collection revealed that subjects reported rating each probe tone as to "how good it sounded" as a completion-that is, as the instructions requested. However, several subjects reported that they could also have rated "how correct it was" according to music theory. We tested this claim with 2 very highly trained musicians operating under two different instructional sets.

The subjects were 2 graduate students in their 20 s, one (D.S.) studying computing and information science, the other (W.F.T.) psychology. Neither had participated in Experiment 2, but both had extensive experience with the probe-tone technique. Their professional musical experience, in composition and piano performance, respectively, was considerably greater than that of the members of the highly trained group.

The subjects were tested with six blocks of the diminished-triad stimulus conditions of the second experiment. Two blocks were practice and two were assigned to each of two instructional sets. One set, called the "grouping" set, instructed the subjects to rate the probe tone as to how good it sounded, or how well it fit the pattern as a group or Gestalt. The other set instructed them to produce the correct music-theoretical response. The order of the two sets was reversed for the 2 subjects.

Under the grouping instructions, both subjects gave the highest rating (1.0) to the probe tones $B$ and $F$. Their second choices were inconsistent. Under the musictheoretical instructions, D.S. gave the highest rating (1.0) to $\mathrm{C}$ and $\mathrm{E}$, followed by $\mathrm{G}$ (2.5); W.F.T. gave the highest rating (1.0) to $\mathrm{C}$, followed by $\mathrm{F}$ and $\mathrm{G}(2.0)$. Fourier analysis was conducted on the profiles, and the profiles were resynthesized from the third and fifth partials. For the grouping instructions, the correlations between the obtained and resynthesized profiles were 0.43 for D.S. and 0.37 for W.F.T. Neither correlation is significant. For the music-theoretical instructions, the correlations between the original and resynthesized profiles were 0.77 for D.S. and 0.94 for W.F.T. ( $p<.01$ for both). These findings suggest that the application of formal musical knowledge makes it possible to recover a tonally strong profile for the diminished triad pattern.

\section{GENERAL DISCUSSION}

The children's profile and the adults' profile for the melodic major triad showed full recovery of the tonal hierarchy and close correspondence with the major-key profiles obtained from triadic chords and chord cadences by 
Krumhansl and Kessler (1982). The efficacy of the major triad and its superiority to the major scale, for both children and adults, is compatible with both Schenkerian approaches in music theory and psychoacoustic approaches that emphasize the role of acoustic consonance in the derivation of the Western tonal system.

In addition, the results are compatible with statistical approaches that enumerate the relative frequency of the major triad as a due to key in music. The tonic triad is favored in the opening of melodic themes, according to the descriptive statistics provided for 15,618 classical themes by Simonton (1984). However, it is unlikely that the children in our study had much exposure to this repertoire. Extended discussions with their school music teacher revealed that a major problem for his pupils was pitch matching. He therefore focused their early lessons not on learning songs, but on pitch matching to piano tones, pitch matching at the octave to the teacher's singing voice, and copying simple patterns.

In any case, recovery of the tonal hierarchy from the major triad was clearly not related to the extent of formal theoretical training. Even the youngest children could decide on a tonic note and could differentiate the "belongingness" of other notes to that tonic. By implication, their profiles also contained the cyclic structure of key relationships. Other evidence-such as the emergence of coherent song patterns and sensitivity to key change in melody recognition-has led to the conclusion that by age 5 or 6 , children have sufficient maturity and cognitive skills to abstract a sense of key (Dowling \& Harwood, 1986, chap. 5).

Both children's and adults' profiles were in agreement in that, after the major triad, the order in which tonal structure was retrieved from stimulus patterns was the major scale followed by the diminished triad. The adults differed from the children, however, in two important ways. First, the adults' profile for the major scale showed greater emphasis on underlying tonal structure and less influence of pitch proximity. The stepwise motion of the scale around the chroma circle appears to have been a more salient feature of the pattern for the children than for the adults. Second, for the diminished-triad pattern, the adults picked up melodic features that the children did not. These were not, however, the intervallic properties that logically implicate a major diatonic key. Only with the specialized instructions in the supplementary experiment-the music-theoretical instructions-did the hierarchy logically implicated by the intervallic structure emerge.

Other evidence has suggested a shift in the salience of melodic features with development (Bartlett \& Dowling, 1980; Trehub, Morrongiello, \& Thorpe, 1985). Trehub et al. asked children and adults to recognize familiar tunes under transformation. Both children and adults rejected contour violations (changes in interval direction) and rejected transformations that preserved the contour but altered the component intervals. But, for the transformations of "Happy Birthday," for example, children were less ready than adults to reject a contour-preserving trans- formation, even though the intervals were changed. They appeared to have been influenced by the overall shape of the melody, an influence that may be similar to that of pitch proximity in the children's profile for the major scale in the present study.

Overall, the data provide further evidence that both adults and children easily and readily acquire intuitive knowledge of the basic tonal structure of music. The major triad appears to be a prototype and a primordial unit of tonal structure. When patterns deviate from this prototype, they introduce other melodic features and, therefore, tonal ambiguities. To resolve the ambiguities, a context must be supplied, and musical knowledge can supply this context. Some of this knowledge is acquired simply through musical exposure or by the development of adult cognitive skills. Other knowledge, such as the correct diatonic solution of the diminished triad, must be formally acquired.

\section{REFERENCES}

Balzano, G. J. (1982). The pitch set as a level of description for studying musical pitch perception. In M. Clynes (Ed.), Music, mind and brain: The neuropsychology of music. New York: Plenum Press.

Balzano, G. J. (1986). What are musical pitch and timbre? Music Perception, 3, 297-314.

Bartlett, J. C., \& Dowling, W. J. (1980). The recognition of transposed melodies: A key-distance effect in developmental perspective. Journal of Experimental Psychology: Human Perception \& Performance, 6, 501-515.

BHARUCHA, J. J. (1984). Anchoring effects in music-the resolution of dissonance. Cognitive Psychology, 16, 485-519.

BrowN, H., \& BUTLER, D. (1981). Diatonic trichords as minimal tonal cue-cells. In Theory Only, 5(6\&7), 39-55.

Brown, M. (1986). The diatonic and the chromatic in Schenker's theory of harmonic relations. Journal of Music Theory, 30, 1-33.

Brown, R. (1981). Tonal implications of the diatonic set. In Theory Only, 5(6\&7), 3-21.

Butler, D., \& Brown, H. (1984). Tonal structure versus function: Studies of the recognition of harmonic motion. Music Perception, 2, 6-24.

Cudpy, L. L. (1985). The color of melody. Music Perception, 2, 345-360.

CudDY, L. L. (1986). The harmony of melody. Proceedings of the 12 th International Congress of Acoustics, Paper K5-1. Toronto: International Congress of Acoustics.

Cuddy, L. L., Cohen, A. J., \& Mewhort, D. J. K. (1981). Perception of structure in short melodic sequences. Journal of Experimental Psychology: Human Perception \& Performance, 7, 869-883.

Cuddy, L. L., Cohen, A. J., \& Miller, J. (1979). Melody recognition: The experimental application of musical rules. Canadian Journal of Psychology, 33, 148-157.

DEUTSCH, D. , \& FEROE, J. (1981). The internal representation of pitch sequences in tonal music. Psychological Review, 88, 503-522.

Dewar, K. M. (1974). Context effects in recognition memory for tones. Unpublished doctoral dissertation, Queen's University, Kingston, Ontario.

Dewar, K. M., Cuddy, L. L., \& Mewhort, D. J. K. (1977). Recognition memory for single tones with and without context. Journal of Experimental Psychology: Human Learning \& Memory, 3, 60-67. Dowling, W. J. (1978). Scale and contour: Two components of a theory of memory for melodies. Psychological Review, 85, 341-354.

Dowling, W. J., \& HaRwood, D. L. (1986). Music cognition. New York: Academic Press.

Jenkins, G. M., \& Watts, D. G. (1968). Spectral analysis and its ap plications. San Francisco: Holden-Day.

FrancÈs, R. (1958). La perception de la musique. Paris: Vrin. 
KrumhansL, C. L. (1979). The psychological representation of musical pitch in a tonal context. Cognitive Psychology, 11, 346-374.

KrumhansL, C. L. (1982). A quantitative description of musical key structure. Paper presented at the meeting of the Society for Mathematical Psychology, Princeton, NJ.

Krumhanst, C. L. (1983). Perceptual structures for tonal music. Music Perception, 1, 28-62.

KrumhansL, C. L. (1986). General properties of musical pitch systems: Some psychological considerations. In J. Sundberg (Ed.), Harmony and tonality. Stockholm: Swedish Academy of Music.

KrumhansL, C. L., \& KeIL, F. C. (1982). Acquisition of the hierarchy of tonal functions in music. Memory \& Cognition, 10, 243-251.

Krumhansl, C. L., \& KeSsler, E. J. (1982). Tracing the dynamic changes in perceived tonal organization in a spatial representation of musical keys. Psychological Review, 89, 334-368.

Krumhansl, C. L., \& Schmuckler, M. A. (1986). Key-finding in music: An algorithm with applications to Bach, Shostakovitch, and Chopin. Unpublished manuscript, Cornell University, Department of Psychology, Ithaca, NY.

KrumhansL, C. L., \& ShePard, R. N. (1979). Quantification of the hierarchy of tonal functions within a diatonic context. Journal of Experimental Psychology: Human Perception \& Performance, 5, 579-594.

Longuet-Higgins, H. C., \& Steedman, M. J. (1971). On interpreting Bach. Machine Intelligence, 6, 221-241.

MAKEIG, S. (1982). Affective versus analytic perception of musical intervals. In M. Clynes (Ed.), Music, mind and brain: The neuropsychology of music. New York: Plenum Press.
Patterson, R. D. (1986). Spiral detection of periodicity and the spiral form of musical scales. Psychology of Music, 14, 44-61.

Piston, W. (1962). Harmony (3rd ed.). New York: W. W. Norton. RAtner, L. G. (1962). Harmony-Structure and style. New York: McGraw-Hill.

Rosch, E. (1975). Cognitive reference points. Cognitive Psychology, 7, 532-547.

Schenker, H. (1954). Harmony (O. Jones, Ed., \& E. M. Borgese, Trans.). Cambridge, MA: MIT Press. (Original work published 1906) Schoenberg, A. (1969). Structural functions of harmony (rev. ed.). New York: Norton. (Original work published 1954)

SHEPARD, R. N. (1964). Circularity in judgments of relative pitch. Journal of the Acoustical Society of America, 36, 2346-2353.

SHEPARD, R. N. (1982). Structural representations of musical pitch. In D. Deutsch (Ed.), The psychology of music. New York: Academic Press.

Simonton, D. K. (1984). Melodic structure and note transition probabilities: A content analysis of 15,618 classical themes. Psychology of Music, 12, 3-6.

SPEER, J. R., \& MEEKS, P. U. (1985). School children's perception of pitch in music. Psychomusicology, 5, 49-56.

TERHARDT, E. (1984). The concept of musical consonance: A link between music and psychoacoustics. Music Perception, 1, 276-295.

Trehub, S. E., Morrongiello, B. A., \& Thorpe, L. A. (1985). Children's perception of familiar melodies: The role of intervals, contour and key. Psychomusicology, 5, 39-48.

WallRafF, D. (1979). The DMX-1000 signal processing computer. Computer Music Journal, 3, 44-49. 